\title{
Cationic gold(I) axially chiral biaryl bisphosphine complex-catalyzed atropselective synthesis of heterobiaryls
}

\author{
Tetsuro Shibuya, Kyosuke Nakamura and Ken Tanaka*
}

\section{Full Research Paper}

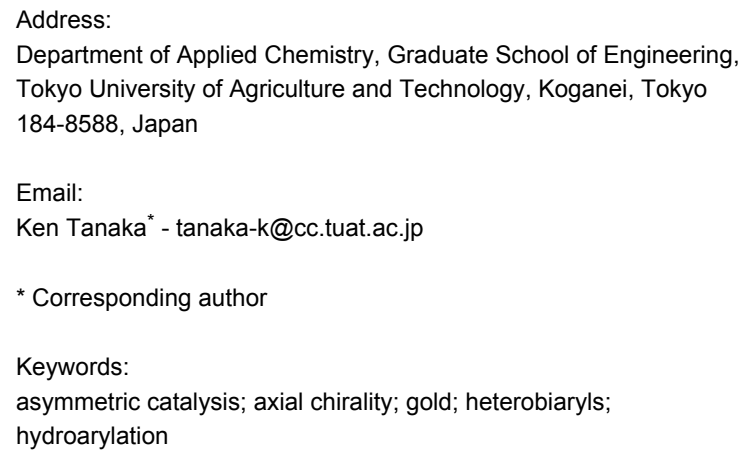

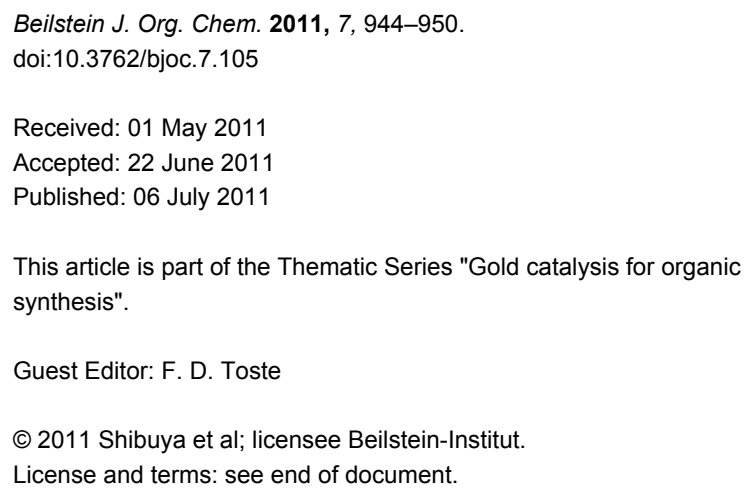

\begin{abstract}
It has been established that a cationic gold(I)/(R)-DTBM-Segphos or $(R)$-BINAP complex catalyzes the atropselective intramolecular hydroarylation of alkynes leading to enantioenriched axially chiral 4-aryl-2-quinolinones and 4-arylcoumarins with up to $61 \%$ ee.
\end{abstract}

\section{Introduction}

Atropselective biaryl synthesis [1-4] has attracted significant interest due to its great utility in asymmetric catalysis and natural product synthesis. In 2004, three research groups, including ours, independently reported transition-metal catalyzed asymmetric $[2+2+2]$ cycloaddition reactions to produce axially chiral biaryls [5-7]. These reports clearly demonstrated the utility of the asymmetric annulation strategy for the atropselective biaryl synthesis [8]. As an alternative asymmetric annulation method for the atropselective biaryl synthesis, we turned our attention to transition-metal catalyzed hydroalkenylation and hydroarylation reactions [9-15]. In this context, our research group developed the cationic gold(I)/ $\mathrm{PPh}_{3}$-complex catalyzed intramolecular hydroalkenylation of
$\mathrm{N}$-alkenyl-arylethynylamides leading to 4-aryl-2-pyridones (Scheme 1) $[16,17]$.



Scheme 1: Cationic gold(I)/PPh 3 -complex catalyzed intramolecular hydroalkenylation of alkynes. 
The atropselective synthesis of 6-aryl-2-pyridones has already been achieved by rhodium catalyzed $[2+2+2]$ cycloaddition [18], while the atropselective synthesis of 4-aryl-2-pyridones has not yet been realized. The application of this intramolecular hydroalkenylation reaction to the atropselective synthesis of 4-aryl-2-pyridones from $\mathrm{N}$-alkenyl-arylethynylamides was thus investigated. Although cationic gold(I)/axially chiral biaryl bisphosphine complexes [19-31] have been frequently employed in asymmetric variants of cationic gold(I) catalyses [32-38], including 6-endo-dig and 6-exo-dig cyclizations [39$41]$, the use of these gold(I) complexes gave almost racemic products [42]. Fortunately, cationic palladium(II)/axially chiral biaryl bisphosphine complexes were found to be effective catalysts, and a cationic palladium(II)/(S)-xyl-Segphos complex showed the highest enantioselectivity (Scheme 2) [42].



$96 \%, 94 \%$ ee

Scheme 2: Cationic palladium(II)/(S)-xyl-Segphos-complex catalyzed atropselective intramolecular hydroalkenylation of alkynes.

In addition, the cationic palladium(II)/axially chiral biaryl bisphosphine complexes were able to catalyze the asymmetric intramolecular hydroarylation of $\mathrm{N}$-aryl-arylethynylamides



$94 \%, 92 \%$ ee

Scheme 3: Cationic palladium(II)/(S)-xyl- $\mathrm{H}_{8}$-BINAP complex-catalyzed atropselective intramolecular hydroarylation of alkynes.

leading to axially chiral 4-aryl-2-quinolinones, and the cationic palladium(II)/(S)-xyl-H 8 -BINAP complex showed the highest enantioselectivity (Scheme 3) $[43,44]$.

In this paper, we report the use of the cationic gold(I)/axially chiral biaryl bisphosphine complexes in the catalytic asymmetric intramolecular hydroarylation for the synthesis of axially chiral 4-aryl-2-quinolinones and 4-arylcoumarins.

\section{Results and Discussion}

The reaction of $N$-benzyl- $N$-(2-naphthyl)propiolamide $\mathbf{1 a}$, bearing a 2-methoxynaphthyl group at an alkyne terminus, was first investigated in the presence of a cationic $\operatorname{gold}(\mathrm{I}) /(R)$ BINAP complex $(20 \mathrm{~mol} \% \mathrm{Au})$. Although the reaction proceeded at room temperature in good yield, enantioselectivity was low (Table 1, entry 1). The effect of axially chiral biaryl bisphosphine ligands (Figure 1) on the yield and the enantioselectivity was then investigated. Among the

Table 1: Screening of axially chiral biaryl bisphosphine ligands for the cationic gold(I)-complex catalyzed atropselective intramolecular hydroarylation of $1 a^{a}$



$1 \mathbf{a}$

$2 \mathbf{a}$

\begin{tabular}{lllll}
\hline Entry & Ligand & Convn $(\%)^{\mathrm{b}}$ & ${\text { Yield }(\%)^{\mathrm{c}}}^{\mathrm{c}}$ & \\
\hline 1 & $(R)$-BINAP & 81 & 75 & $7(S)$ \\
2 & $(R)$-Segphos & 90 & 81 & $8(R)$ \\
3 & $(R)$-H -BINAP & 94 & 93 & $17(S)$ \\
4 & $(S)$-xyl-H8-BINAP & 100 & 96 & $5(R)$ \\
5 & $(R)$-DTBM-Segphos & 100 & 96 & $59(R)$ \\
$6^{\text {d }}$ & $(R)$-DTBM-Segphos & 71 & 49 & $31(R)$ \\
\hline
\end{tabular}

${ }^{\mathrm{a}} \mathrm{AuCl}\left(\mathrm{SMe}_{2}\right)(0.010 \mathrm{mmol}, 20 \mathrm{~mol} \%), \mathrm{AgBF}_{4}(0.010 \mathrm{mmol}, 20 \mathrm{~mol} \%)$, ligand $(0.0050 \mathrm{mmol}, 10 \mathrm{~mol} \%), 1 \mathrm{a}(0.050 \mathrm{mmol})$, and $\left(\mathrm{CH} \mathrm{Cl}_{2}(1.5 \mathrm{~mL})\right.$ were used. ${ }^{b}$ Determined by ${ }^{1} \mathrm{H}$ NMR. CIsolated yield. ${ }^{\mathrm{d}} \mathrm{AuCl}\left(\mathrm{SMe}_{2}\right)(0.010 \mathrm{mmol}, 10 \mathrm{~mol} \%), \mathrm{AgBF}_{4}(0.010 \mathrm{mmol}, 10 \mathrm{~mol} \%)$, ligand $(0.0050 \mathrm{mmol}, 5$ $\mathrm{mol} \%), 1 \mathrm{a}(0.10 \mathrm{mmol})$, and $\left(\mathrm{CH}_{2} \mathrm{Cl}\right)_{2}(1.5 \mathrm{~mL})$ were used. Reaction time: $72 \mathrm{~h}$. 
<smiles>Pc1ccc2ccccc2c1-c1c(P(c2ccccc2)c2ccccc2)ccc2ccccc12</smiles>

(R)-BINAP

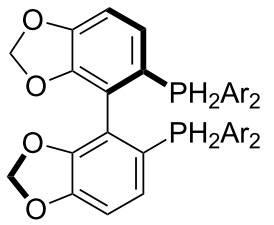

$(R)$-Segphos $(\mathrm{Ar}=\mathrm{Ph})$ $(R)$-DTBM-Segphos $\left(\mathrm{Ar}=4-\mathrm{MeO}^{-3}, 5-t-\mathrm{Bu}_{2} \mathrm{C}_{6} \mathrm{H}_{2}\right)$<smiles>Pc1ccc2c(c1-c1c(P)ccc3c1CCCC3)CCCC2</smiles>

$(R)-\mathrm{H}_{8}$-BINAP $(\mathrm{Ar}=\mathrm{Ph})$ $(R)-\mathrm{xyl}-\mathrm{H}_{8}$-BINAP $\left(\mathrm{Ar}=3,5-\mathrm{Me}_{2} \mathrm{C}_{6} \mathrm{H}_{3}\right)$

Figure 1: Structures of axially chiral biaryl bisphosphine ligands

bis(diphenylphosphine) ligands examined (Table 1, entries $1-3)$, the use of $(R)-\mathrm{H}_{8}$-BINAP furnished $\mathbf{2 a}$ with the highest enantiomeric excess (Table 1, entry 3 ). An increase in the steric bulk of the aryl group on the phosphorus atom of $\mathrm{H}_{8}$-BINAP lead to a decrease in the ee (Table 1, entry 4$)$. The use of sterically more demanding $(R)$-DTBM-Segphos as a ligand furnished 2a in high yield with the highest ee (Table 1, entry 5). Unfortunately, a reduction in the amount of gold to $10 \mathrm{~mol} \%$ significantly decreased both product yield and enantioselectivity (Table 1, entry 6).

Thus, the scope of the cationic gold(I)-complex catalyzed atropselective intramolecular hydroarylation of alkynes was explored at room temperature, as shown in Table 2. The 2-methoxynaphthalene derivative 1a (Table 2, entry 1) and the 2-methoxymethoxynaphthalene derivative $\mathbf{1 b}$ (Table 2, entry 2 ) furnished the desired benzoquinolinones $\mathbf{2 a}$ and $\mathbf{2 b}$, respectively, in high yields and high ee, using ( $R$ )-DTBM-Segphos as a ligand. In addition, benzocoumarin $2 \mathrm{c}$ (Table 2, entry 3 ) was obtained in moderate ee, although the yield was low due to partial deprotection of the methoxymethoxynaphthalene moiety (Table 2, entry 3 ). The reactions of carbazole and dialkoxybenzene derivatives 1d-g, using ( $R$ )-DTBM-Segphos as a ligand, furnished the corresponding quinolinone and coumarin derivatives $\mathbf{2 d -}-\mathbf{g}$ in high yields with perfect regioselectivity, while the observed ee values were very low $(<10 \%$ ee). However, interestingly, the use of $(R)$-BINAP as a ligand improved the enantoselectivity (14-32\% ee, Table 2, entries 4-7).

Table 2: Cationic gold(I)-complex catalyzed atropselective intramolecular hydroarylation of $\mathbf{1 a}-\mathbf{g}$ leading to heterobiaryls $\mathbf{2 a - g} .^{\text {a }}$

$\begin{array}{llll}\text { Entry } & \text { Ligand } & \mathbf{2} & \text { (time) }\end{array}$

1<smiles>COc1ccc2ccccc2c1C#CC(=O)N(Cc1ccccc1)c1ccc2ccccc2c1</smiles>

2<smiles>COc1ccc2ccccc2c1C#CC(=O)N(Cc1ccccc1)c1ccc2ccccc2c1</smiles>

1b Segphos (72 h)

(R)-DTBM-

1a Segphos (40 h)<smiles>COc1ccc2ccccc2c1-c1cc(=O)n(Cc2ccccc2)c2ccc3ccccc3c12</smiles>

$(R)-(-)-2 a$

96 (59)

$\mathrm{Bn}$<smiles>COc1ccc2ccccc2c1-c1cc(=O)n(Cc2ccccc2)c2ccc3ccccc3c12</smiles>

$(-)-2 b$

87 (61)
(R)-DTBM-

1c Segphos (40 h)

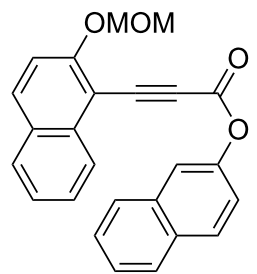<smiles>COc1ccc2ccccc2c1-c1cc(=O)oc2ccc3ccccc3c12</smiles> 
Table 2: Cationic gold(I)-complex catalyzed atropselective intramolecular hydroarylation of $\mathbf{1 a - g}$ leading to heterobiaryls $\mathbf{2 a - \mathbf { g }}{ }^{\text {a }}$ (continued)

4<smiles>CCn1c2ccccc2c2cc(N(Cc3ccccc3)C(=O)C#Cc3c(OC)ccc4ccccc34)ccc21</smiles>

5<smiles>COc1cc(OC)cc(N(Cc2ccccc2)C(=O)C#Cc2c(C)cccc2OC)c1</smiles>

6<smiles>COc1ccc2ccccc2c1C#CC(=O)N(Cc1ccccc1)c1ccc2c(c1)OCO2</smiles>

7



(R)-BINAP

(72 h)<smiles></smiles>

$\mathrm{I}$
$\mathrm{Bn}$<smiles>COc1cc(OC)c2c(-c3c(C)cccc3OC)cc(=O)n(Cc3ccccc3)c2c1</smiles>

(R)-BINAP (40 h)<smiles>COc1ccc2ccccc2c1-c1cc(=O)n(Cc2ccccc2)c2cc3c(cc12)OCO3</smiles>

(R)-BINAP (40 h) (+)-2f

(+)-2d

(+)-2g

93 (14)

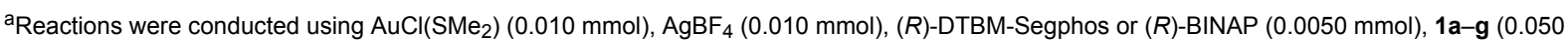
$\mathrm{mmol})$, and $\left(\mathrm{CH}_{2} \mathrm{Cl}\right)_{2}(1.5 \mathrm{~mL})$ at rt. In all entries, $100 \%$ convn of substrates $\mathbf{1 a}-\mathbf{g}$ was observed.

bIsolated yield.

In the previously reported cationic palladium(II)/(S)-xyl- $\mathrm{H}_{8}-$ BINAP-complex catalyzed atropselective intramolecular hydroarylation of alkynes, the presence of the 2-methoxysubstituted aryl group at the alkyne terminus was important for the realization of both high reactivity and enantioselectivity [40]. Similarly, the reaction of 2-methylnaphthalene derivative $\mathbf{1 h}$ in the presence of the cationic gold(I)/(R)-DTBM-Segphos complex furnished the corresponding benzoquinolinone $\mathbf{2 h}$ with lower yield and ee than those of $\mathbf{2 a}$ (Scheme 4).

\section{Conclusion}

In conclusion, it has been established that a cationic $\operatorname{gold}(\mathrm{I}) /(R)-$ DTBM-Segphos or $(R)$-BINAP complex catalyzes the atropselective intramolecular hydroarylation of alkynes leading to

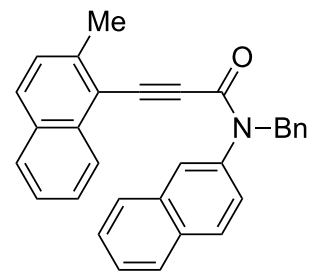

$1 \mathrm{~h}$
$20 \mathrm{~mol} \% \mathrm{AuCl}\left(\mathrm{SMe}_{2}\right)$ $10 \mathrm{~mol} \%(R)$-DTBM-Segphos $20 \mathrm{~mol}_{\%} \mathrm{AgBF}_{4}$

$\left(\mathrm{CH}_{2} \mathrm{Cl}\right)_{2}, \mathrm{rt}, 72 \mathrm{~h}$

(93\% convn)<smiles>Cc1ccc2ccccc2c1-c1cc(=O)n(Cc2ccccc2)c2ccc3ccccc3c12</smiles>

(+)-2h / 62\%, 15\% ee

Scheme 4: Cationic gold(I)/(R)-DTBM-Segphos-complex catalyzed atropselective intramolecular hydroarylation of 2-methylnaphthalene derivative 1h 
enantioenriched axially chiral 4-aryl-2-quinolinones and 4-arylcoumarins in up to $61 \%$ ee. Although there clearly remains room for improvement in enantioselectivity, the present asymmetric catalysis is a rare example of the utilization of gold(I)/ chiral phosphine catalysts for the construction of noncentrochirality [45-47].

\section{Experimental}

General: ${ }^{1} \mathrm{H}$ NMR spectra were recorded at $300 \mathrm{MHz}$ (JEOL AL 300). ${ }^{13} \mathrm{C}$ NMR spectra were obtained with complete proton decoupling at $75 \mathrm{MHz}$ (JEOL AL 300). HRMS data were obtained on a Bruker micrOTOF Focus II. Infrared spectra were obtained on a JASCO FT/IR-4100. Optical rotations were obtained on a JASCO DIP-1000. Melting points were obtained on a METTLER MP50. Anhydrous $\left(\mathrm{CH}_{2} \mathrm{Cl}\right)_{2}$ (No. 28,450-5) was purchased from Aldrich and used as received. Solvents for the synthesis of substrates were dried over molecular sieves (4 $\AA$, Wako) prior to use. Substrates $\mathbf{1 a}, \mathbf{1 b}, \mathbf{1 d}, \mathbf{1 e}, \mathbf{1 f}$, and $\mathbf{1 h}$ were prepared according to the literature [43]. Products $\mathbf{2 a}, \mathbf{2 b}$, $\mathbf{2 d}, \mathbf{2 e}, \mathbf{2 f}$, and $\mathbf{2 h}$ were already reported [43]. All other reagents were obtained from commercial sources and used as received. All reactions were carried out under an atmosphere of argon or nitrogen in oven-dried glassware with magnetic stirring.

(2-Methoxymethoxynaphthalen-1-yl)propynoic acid naphthalen-2-yl ester (1c): To a stirred solution of 3-[2(methoxymethoxy)-1-naphthalenyl]-2-propynoic acid [48] (0.256 g, $1.00 \mathrm{mmol})$, 2-naphthol $(0.159 \mathrm{~g}, 1.10 \mathrm{mmol})$, and 4-dimethylaminopyridine $(12.2 \mathrm{mg}, 0.100 \mathrm{mmol})$ in $\mathrm{CH}_{2} \mathrm{Cl}_{2}(10$ $\mathrm{mL})$ was added a solution of dicyclohexylcarbodiimide $(0.248$ $\mathrm{g}, 1.20 \mathrm{mmol})$ in $\mathrm{CH}_{2} \mathrm{Cl}_{2}(3 \mathrm{~mL})$ at $0{ }^{\circ} \mathrm{C}$, and the mixture was stirred at $0{ }^{\circ} \mathrm{C}$ for $2 \mathrm{~h}$ and at room temperature for $18 \mathrm{~h}$. The crude mixture was filtered with $\mathrm{CH}_{2} \mathrm{Cl}_{2}$. The filtrate was washed with brine, dried over $\mathrm{Na}_{2} \mathrm{SO}_{4}$, and concentrated. The residue was purified by a silica gel column chromatography (hexane/EtOAc $=10: 1)$ to give $1 \mathrm{c}(0.222 \mathrm{~g}, 0.580 \mathrm{mmol}, 58 \%$ yield). Yellow solid; mp 97.3-99.3 ${ }^{\circ} \mathrm{C}$; IR (KBr): 2203, 1717, 1229, 1149, $1005 \mathrm{~cm}^{-1} ;{ }^{1} \mathrm{H} \mathrm{NMR}\left(\mathrm{CDCl}_{3}, 300 \mathrm{MHz}\right) \delta$ 8.15-8.01 (m, 1H), 7.97-7.70 (m, 6H), 7.61-7.31 (m, 6H), 5.36 $(\mathrm{s}, 2 \mathrm{H}), 3.56(\mathrm{~s}, 3 \mathrm{H}) ;{ }^{13} \mathrm{C} \mathrm{NMR}\left(\mathrm{CDCl}_{3}, 125 \mathrm{MHz}\right) \delta 160.0$, 152.8, 148.1, 134.7, 133.7, 133.4, 131.7, 129.7, 128.8, 128.3, $127.82,127.79,126.7,126.0,125.1,124.8,121.0,118.8,115.5$, 103.8, 95.1, 89.1, 85.2, 56.6; HRMS-ESI $(\mathrm{m} / \mathrm{z}):[\mathrm{M}+\mathrm{Na}]^{+}$ calcd for $\mathrm{C}_{25} \mathrm{H}_{18} \mathrm{O}_{4} \mathrm{Na}$, 405.1097; found, 405.1107 .

(2-Methoxynaphthalen-1-yl)propynoic acid 3,5-dimethoxyphenyl ester (1g): The title compound was prepared from (2-methoxynaphthalen-1-yl)propynoic acid [49] and 3,5dimethoxyphenol in $70 \%$ yield by the procedure used for $\mathbf{1 c}$ Yellow solid; mp 102.9-104.7 ${ }^{\circ} \mathrm{C}$; IR (KBr): 2211, 1714, 1621, $1269,1156 \mathrm{~cm}^{-1} ;{ }^{1} \mathrm{H} \mathrm{NMR}\left(\mathrm{CDCl}_{3}, 300 \mathrm{MHz}\right) \delta 8.16(\mathrm{~d}, J=$
$8.4 \mathrm{~Hz}, 1 \mathrm{H}), 7.96(\mathrm{~d}, J=9.2 \mathrm{~Hz}, 1 \mathrm{H}), 7.80(\mathrm{~d}, J=8.1 \mathrm{~Hz}, 1 \mathrm{H})$, $7.59(\mathrm{dd}, J=8.1,6.5 \mathrm{~Hz}, 1 \mathrm{H}), 7.42(\mathrm{dd}, J=8.4,6.5 \mathrm{~Hz}, 1 \mathrm{H})$, $7.26(\mathrm{~d}, J=9.2 \mathrm{~Hz}, 1 \mathrm{H}), 6.43(\mathrm{~d}, J=2.1 \mathrm{~Hz}, 2 \mathrm{H}), 6.40(\mathrm{t}, J=$ $2.1 \mathrm{~Hz}, 1 \mathrm{H}), 4.06(\mathrm{~s}, 3 \mathrm{H}), 3.80(\mathrm{~s}, 6 \mathrm{H}) ;{ }^{13} \mathrm{C} \mathrm{NMR}\left(\mathrm{CDCl}_{3}, 125\right.$ $\mathrm{MHz}) \delta 162.1,161.1,152.5,151.8,135.0,133.6,128.4,128.3$, 128.1, 124.7, 112.1, 101.8, 100.2, 98.7, 89.3, 85.0, 56.5, 55.5; HRMS-ESI $(\mathrm{m} / \mathrm{z}):[\mathrm{M}+\mathrm{Na}]^{+}$calcd for $\mathrm{C}_{22} \mathrm{H}_{18} \mathrm{O}_{5} \mathrm{Na}, 385.1046$; found, 385.1047 .

General procedure for cationic gold(I)/axially chiral biaryl bisphosphine complex-catalyzed atropselective intramolecular hydroarylation of $\mathbf{N}$-aryl-arylethynylamides 1 : To $\mathrm{AuCl}\left(\mathrm{SMe}_{2}\right)(0.010 \mathrm{mmol})$ was added a solution of axially chiral biaryl bisphosphine ligand $(0.0050 \mathrm{mmol})$ in $\left(\mathrm{CH}_{2} \mathrm{Cl}\right)_{2}$ $(0.5 \mathrm{~mL})$, and the mixture was stirred at room temperature for 1 h. To this solution was added $\mathrm{AgBF}_{4}(0.010 \mathrm{mmol})$ in $\left(\mathrm{CH}_{2} \mathrm{Cl}\right)_{2}$ $(0.5 \mathrm{~mL})$ at room temperature, and the mixture was stirred at room temperature for $0.5 \mathrm{~h}$. To this mixture was added a solution of $1(0.050 \mathrm{mmol})$ in $\left(\mathrm{CH}_{2} \mathrm{Cl}\right)_{2}(0.5 \mathrm{~mL})$ at room temperature. After stirring at room temperature for $40-72 \mathrm{~h}$, the mixture was directly purified on a preparative TLC to afford $\mathbf{2}$.

(-)-1-(2-Methoxymethoxynaphthalen-1-yl)benzo[f]chromen3-one [(-)-2c]: Colorless solid; mp $169.4-170.8^{\circ} \mathrm{C}$; $[\alpha]^{25} \mathrm{D}$ -86.1 ( c $0.28, \mathrm{CHCl}_{3}, 49 \%$ ee); IR (KBr): 1738, 1510, 1244, $1050,1011 \mathrm{~cm}^{-1} ;{ }^{1} \mathrm{H} \mathrm{NMR}\left(\mathrm{CDCl}_{3}, 500 \mathrm{MHz}\right) \delta 8.05(\mathrm{~d}, J=$ $8.7 \mathrm{~Hz}, 1 \mathrm{H}), 8.02(\mathrm{~d}, J=9.2 \mathrm{~Hz}, 1 \mathrm{H}), 7.92(\mathrm{~d}, J=8.4 \mathrm{~Hz}, 1 \mathrm{H})$, $7.81(\mathrm{~d}, J=8.0 \mathrm{~Hz}, 1 \mathrm{H}), 7.62(\mathrm{~d}, J=8.7 \mathrm{~Hz}, 1 \mathrm{H}), 7.57$ (d, $J=$ $9.2 \mathrm{~Hz}, 1 \mathrm{H}), 7.53(\mathrm{~d}, J=8.4 \mathrm{~Hz}, 1 \mathrm{H}), 7.42(\mathrm{ddd}, J=8.4,7.0$, $1.4 \mathrm{~Hz}, 1 \mathrm{H}), 7.36$ (ddd, $J=8.4,6.9,1.5 \mathrm{~Hz}, 1 \mathrm{H}), 7.32$ (ddd, $J=$ 8.0, 7.0, $1.0 \mathrm{~Hz}, 1 \mathrm{H}), 7.17(\mathrm{~d}, J=8.3 \mathrm{~Hz}, 1 \mathrm{H}), 6.97$ (ddd, $J=$ $8.3,6.9,1.4 \mathrm{~Hz}, 1 \mathrm{H}), 6.45$ (s, $1 \mathrm{H}), 5.04(\mathrm{dd}, J=22.4,6.9 \mathrm{~Hz}$, $2 \mathrm{H}), 3.05(\mathrm{~s}, 3 \mathrm{H}) ;{ }^{13} \mathrm{C} \mathrm{NMR}\left(\mathrm{CDCl}_{3}, 125 \mathrm{MHz}\right) \delta 160.6,154.8$, 152.5, 150.6, 133.8, 131.6, 131.0, 130.9, 129.8, 129.5, 129.0, $128.2,127.7,127.6,125.4,124.8,124.4,123.8,122.8,118.6$, 117.8, 115.7, 114.2, 94.2, 56.0; HRMS-ESI $(\mathrm{m} / \mathrm{z}):[\mathrm{M}+\mathrm{Na}]^{+}$ calcd for $\mathrm{C}_{25} \mathrm{H}_{18} \mathrm{O}_{4} \mathrm{Na}$, 405.1097; found, 405.1085; CHIRALPAK OD-H, hexane $/ \mathrm{iPrOH}=80: 20,1.0 \mathrm{~mL} / \mathrm{min}$, retention times: $14.3 \mathrm{~min}$ (major isomer) and $19.0 \mathrm{~min}$ (minor isomer).

(+)-5,7-Dimethoxy-4-(2-methoxynaphthalen-1-yl)chromen2-one $[(+)-2 g]$ : Colorless solid; $m p ~ 148.8-150.4{ }^{\circ} \mathrm{C} ;[\alpha]^{25} \mathrm{D}$ +44.9 (c 0.24, $\mathrm{CHCl}_{3}, 14 \%$ ee); IR (KBr): 1718, 1618, 1598, $1351,1114 \mathrm{~cm}^{-1} ;{ }^{1} \mathrm{H} \mathrm{NMR}\left(\mathrm{CDCl}_{3}, 300 \mathrm{MHz}\right) \delta 7.88(\mathrm{~d}, J=$ 9.0 Hz, 1H), 7.85-7.77 (m, 1H), 7.48-7.39 (m, 1H), 7.38-7.27 (m, 3H), $6.57(\mathrm{~d}, J=2.4 \mathrm{~Hz}, 1 \mathrm{H}), 6.12(\mathrm{~d}, J=2.4 \mathrm{~Hz}, 1 \mathrm{H}), 6.07$ $(\mathrm{s}, 1 \mathrm{H}), 3.85(\mathrm{~s}, 3 \mathrm{H}), 3.83(\mathrm{~s}, 3 \mathrm{H}) 3.07(\mathrm{~s}, 3 \mathrm{H}) ;{ }^{13} \mathrm{C} \mathrm{NMR}$ $\left(\mathrm{CDCl}_{3}, 125 \mathrm{MHz}\right) \delta 163.1,161.2,158.5,157.1,152.4,151.0$, $131.9,129.3,128.5,127.9,126.6,124.2,123.6,122.9,114.0$, 113.1, 105.0, 95.8, 93.6, 56.7, 55.75, 55.71; HRMS-ESI $(\mathrm{m} / \mathrm{z})$ : 
$[\mathrm{M}+\mathrm{Na}]^{+}$calcd for $\mathrm{C}_{22} \mathrm{H}_{18} \mathrm{O}_{5} \mathrm{Na}, 385.1046$; found, 385.1036; CHIRALPAK AD-H, hexane $/ \mathrm{iPrOH}=80: 20,1.0 \mathrm{~mL} / \mathrm{min}$, retention times: $8.8 \mathrm{~min}$ (minor isomer) and $10.5 \mathrm{~min}$ (major isomer).

\section{Supporting Information}

\section{Supporting Information File 1}

${ }^{1} \mathrm{H}$ and ${ }^{13} \mathrm{C}$ NMR spectra for new compounds $1 \mathrm{c}, \mathbf{1 g}, \mathbf{2 c}$, and $\mathbf{2 g}$.

[http://www.beilstein-journals.org/bjoc/content/ supplementary/1860-5397-7-105-S1.pdf]

\section{Acknowledgements}

This work was supported partly by a Grant-in-Aid for Scientific Research (No. 20675002) from MEXT, Japan. We thank Takasago International Corporation for the donation of Segphos, $\mathrm{H}_{8}$-BINAP, xyl-H - -BINAP, and DTBM-Segphos.

\section{References}

1. Kozlowski, M. C.; Morgan, B. J.; Linton, E. C. Chem. Soc. Rev. 2009, 38, 3193-3207. doi:10.1039/b821092f

2. Wallace, T. W. Org. Biomol. Chem. 2006, 4, 3197-3210. doi:10.1039/b608470m

3. Bringmann, G.; Price Mortimer, A. J.; Keller, P. A.; Gresser, M. J.; Garner, J.; Breuning, M. Angew. Chem., Int. Ed. 2005, 44, 5384-5427. doi:10.1002/anie.200462661

4. Baudoin, O. Eur. J. Org. Chem. 2005, 4223-4229. doi:10.1002/ejoc.200500394

5. Gutnov, A.; Heller, B.; Fischer, C.; Drexler, H.-J.; Spannenberg, A.; Sundermann, B.; Sundermann, C. Angew. Chem., Int. Ed. 2004, 43, 3795-3797. doi:10.1002/anie.200454164

6. Shibata, T.; Fujimoto, T.; Yokota, K.; Takagi, K. J. Am. Chem. Soc. 2004, 126, 8382-8383. doi:10.1021/ja048131d

7. Tanaka, K.; Nishida, G.; Wada, A.; Noguchi, K. Angew. Chem., Int. Ed. 2004, 43, 6510-6512. doi:10.1002/anie.200461533

8. Tanaka, K. Chem.-Asian J. 2009, 4, 508-518. doi:10.1002/asia.200800378

9. Kitamura, T. Eur. J. Org. Chem. 2009, 1111-1125. doi:10.1002/ejoc.200801054

10. Skouta, R.; Li, C.-J. Tetrahedron 2008, 64, 4917-4938. doi:10.1016/j.tet.2008.03.083

11. Shen, H. C. Tetrahedron 2008, 64, 3885-3903. doi:10.1016/j.tet.2008.01.081

12. Bandini, M.; Emer, E.; Tommasi, S.; Umani-Ronchi, A. Eur. J. Org. Chem. 2006, 3527-3544. doi:10.1002/ejoc.200500995

13. Nevado, C.; Echavarren, A. M. Synthesis 2005, 167-182. doi:10.1055/s-2005-861781

14. Kakiuchi, F.; Chatani, N. Adv. Synth. Catal. 2003, 345, 1077-1101. doi:10.1002/adsc.200303094

15. Jia, C.; Kitamura, T.; Fujiwara, Y. Acc. Chem. Res. 2001, 34, 633-639. doi:10.1021/ar000209h

16. Imase, H.; Noguchi, K.; Hirano, M.; Tanaka, K. Org. Lett. 2008, 10 , 3563-3566. doi:10.1021/ol801466f
17. Imase, H.; Tanaka, K. Chem. Lett. 2009, 38, 1152-1153. doi:10.1246/cl.2009.1152

18. Tanaka, K.; Wada, A.; Noguchi, K. Org. Lett. 2005, 7, 4737-4739. doi:10.1021/ol052041b

19. Melhado, A. D.; Amarante, G. W.; Wang, Z. J.; Luparia, M.; Toste, F. D. J. Am. Chem. Soc. 2011, 133, 3517-3527. doi:10.1021/ja1095045

20. LaLonde, R. L.; Wang, Z. J.; Mba, M.; Lackner, A. D.; Toste, F. D. Angew. Chem., Int. Ed. 2010, 49, 598-601. doi:10.1002/anie.200905000

21. Kleinbeck, F.; Toste, F. D. J. Am. Chem. Soc. 2009, 131, 9178-9179. doi:10.1021/ja904055z

22. Zhang, Z.; Lee, S. D.; Widenhoefer, R. A. J. Am. Chem. Soc. 2009, 131, 5372-5373. doi:10.1021/ja9001162

23. Uemura, M.; Watson, I. D. G.; Katsukawa, M.; Toste, F. D. J. Am. Chem. Soc. 2009, 131, 3464-3465. doi:10.1021/ja900155x

24. Watson, I. D. G.; Ritter, S.; Toste, F. D. J. Am. Chem. Soc. 2009, 131, 2056-2057. doi:10.1021/ja8085005

25. Chao, C.-M.; Vitale, M. R.; Toullec, P. Y.; Genêt, J.-P.; Michelet, V. Chem.-Eur. J. 2009, 15, 1319-1323. doi:10.1002/chem.200802341

26. Luzung, M. R.; Mauleón, P.; Toste, F. D. J. Am. Chem. Soc. 2007, 129, 12402-12403. doi:10.1021/ja075412n

27. Tarselli, M. A.; Chianese, A. R.; Lee, S. J.; Gagné, M. R. Angew. Chem., Int. Ed. 2007, 46, 6670-6673. doi:10.1002/anie.200701959

28. Liu, C.; Widenhoefer, R. A. Org. Lett. 2007, 9, 1935-1938. doi:10.1021/ol070483c

29. LaLonde, R. L.; Sherry, B. D.; Kang, E. J.; Toste, F. D. J. Am. Chem. Soc. 2007, 129, 2452-2453. doi:10.1021/ja068819।

30. Zhang, Z.; Widenhoefer, R. A. Angew. Chem., Int. Ed. 2007, 46, 283-285. doi:10.1002/anie.200603260

31. Johansson, M. J.; Gorin, D. J.; Staben, S. T.; Toste, F. D. J. Am. Chem. Soc. 2005, 127, 18002-18003. doi:10.1021/ja0552500

32. Fürstner, A. Chem. Soc. Rev. 2009, 38, 3208-3221. doi:10.1039/b816696j

33. Widenhoefer, R. A. Chem.-Eur. J. 2008, 14, 5382-5391. doi:10.1002/chem.200800219

34. Shen, H. C. Tetrahedron 2008, 64, 7847-7870. doi:10.1016/j.tet.2008.05.082

35. Gorin, D. J.; Sherry, B. D.; Toste, F. D. Chem. Rev. 2008, 108, 3351-3378. doi:10.1021/cr068430g

36. Li, Z.; Brouwer, C.; He, C. Chem. Rev. 2008, 108, 3239-3265. doi:10.1021/cr068434I

37. Gorin, D. J.; Toste, F. D. Nature 2007, 446, 395-403. doi:10.1038/nature05592

38. Hashmi, A. S. K.; Hutchings, G. J. Angew. Chem., Int. Ed. 2006, 45, 7896-7936. doi:10.1002/anie.200602454

39. Chao, C.-M.; Beltrami, D.; Toullec, P. Y.; Michelet, V. Chem. Commun. 2009, 6988-6990. doi:10.1039/b913554e

40. Sethofer, S. G.; Staben, S. T.; Hung, O. Y.; Toste, F. D. Org. Lett. 2008, 10, 4315-4318. doi:10.1021/ol801760w

41. Sethofer, S. G.; Mayer, T.; Toste, F. D. J. Am. Chem. Soc. 2010, 132, 8276-8277. doi:10.1021/ja103544p

42. Imase, H.; Suda, T.; Shibata, Y.; Noguchi, K.; Hirano, M.; Tanaka, K. Org. Lett. 2009, 11, 1805-1808. doi:10.1021/ol900373z

43. Shibuya, T.; Shibata, Y.; Noguchi, K.; Tanaka, K. Angew. Chem., Int. Ed. 2011, 50, 3963-3967. doi:10.1002/anie.201100152

44. Ototake, N.; Morimoto, Y.; Mokuya, A.; Fukaya, H.; Shida, Y.; Kitagawa, O. Chem.-Eur. J. 2010, 16, 6752-6755. doi:10.1002/chem.201000243 
45. Ogasawara, M.; Watanabe, S. Synthesis 2009, 1761-1785. doi:10.1055/s-0029-1216818

46. Michon, C.; Liu, S.; Hiragushi, S.; Uenishi, J.; Uemura, M. Synlett 2008, 1321-1324. doi:10.1055/s-2008-1072614

47. Murai, M.; Uenishi, J.; Uemura, M. Org. Lett. 2010, 12, 4788-4791. doi:10.1021/ol1019376

48. Sato, Y.; Tamura, T.; Kinbara, A.; Mori, M. Adv. Synth. Catal. 2007, 349, 647-661. doi:10.1002/adsc.200600587

49. Wessig, P.; Müller, G. Chem. Commun. 2006, 4524-4526. doi:10.1039/B609374D

\section{License and Terms}

This is an Open Access article under the terms of the Creative Commons Attribution License

(http://creativecommons.org/licenses/by/2.0), which permits unrestricted use, distribution, and reproduction in any medium, provided the original work is properly cited.

The license is subject to the Beilstein Journal of Organic Chemistry terms and conditions:

(http://www.beilstein-journals.org/bjoc)

The definitive version of this article is the electronic one which can be found at: doi:10.3762/bjoc.7.105 